Yann Rollin · Michael Singer

\title{
Construction of Kähler surfaces with constant scalar curvature
}

\author{
For Professor Paul Gauduchon on his 60th birthday
}

Received October 10, 2007

Abstract. We present new constructions of Kähler metrics with constant scalar curvature on complex surfaces, in particular on certain del Pezzo surfaces. Some higher-dimensional examples are provided as well.

\section{Introduction}

The aim of this note is to present a new construction of Kähler metrics of constant scalar curvature (CSC) on complex surfaces. In order to introduce our results, let us introduce the terms "positive CSC" to mean "constant positive scalar curvature", "zero CSC" for "(constant) zero scalar curvature" and "negative CSC" for "constant negative scalar curvature".

Our construction gives rise to many families of examples, but in this introduction we shall focus on

$$
X_{k}:=k \text {-fold blow-up of } \mathbb{C P}^{1} \times \mathbb{C P}^{1} .
$$

We note that if $k \geq 1$ then $X_{k}$ can also be viewed as a $k+1$-fold blow-up of $\mathbb{C P}^{2}$. Of course, the above description of $X_{k}$ does not fix its complex structure: this will depend on the location of the centres of the blow-ups. Our first result gives positive CSC Kähler metrics in a family of Kähler classes on $X_{k}$, for $k=6,7,8$, and for certain choices of complex structure. We note that if $k \leq 7$ then $X_{k}$ is Fano and the work of Tian [13] and others gives positive Kähler-Einstein metrics on $X_{k}$. Our result is new in that it produces CSC metrics on $X_{8}$ as well as CSC metrics on $X_{6}$ and $X_{7}$ in Kähler classes that are "arbitrarily far" from $c_{1}(X)$ :

Theorem A. For $k=6,7,8$, there exists a $k$-point blow-up $X$ of $\mathbb{C P}^{1} \times \mathbb{C P}^{1}$ with no non-trivial holomorphic vector field and the following properties. Let $F=\{x\} \times \mathbb{C P}^{1}$ be a generic rational curve of $\mathbb{C P}^{1} \times \mathbb{C P}^{1}$. For every constant $c>0$ and $\varepsilon>0$, there exists a Kähler metric $\omega$ of strictly positive constant scalar curvature on $X$ such that

$$
\left|\frac{[\omega] \cdot F}{\sqrt{[\omega]^{2}}}-c\right| \leq \varepsilon .
$$

Y. Rollin: Imperial College, London, England; e-mail: rollin@imperial.ac.uk

M. Singer: University of Edinburgh, Scotland; e-mail: michael@ maths.ed.ac.uk 
This theorem can be thought of as an exploration of the Kähler cone on certain del Pezzo surfaces. We are asking the question: which Kähler classes can be realized by Kähler metrics of strictly positive constant scalar curvature?

As we shall see later in Section 2 and Theorem C, where the tools used to construct the metrics provided by Theorem A are explained, one can pin down the Kähler class of the metrics up to a parameter $\varepsilon$. The inequality (1.1) is just a consequence of this fact. The parameter is actually involved in the Arezzo-Pacard gluing theorem [1] used to produce the metrics.

Since the geometric interpretation of the Kähler class expressed in an arbitrary basis would be unclear, we prefer to give a formula (up to $\varepsilon$ ) later (cf. Section 2.2), once a suitable formalism has been introduced.

Remark. For the KE metric on $X_{k}, k=6,7$, the number $c$ takes the value

$$
c^{\mathrm{ein}}:=\frac{\left[\omega^{\mathrm{ein}}\right] \cdot F}{\sqrt{\left[\omega^{\mathrm{ein}}\right]^{2}}}=\frac{c_{1}(X) \cdot F}{\sqrt{c_{1}^{2}(X)}}=\frac{2}{\sqrt{8-k}} .
$$

Remark. It is an observation of LeBrun (see, for example, [9]) that the hyperplane

$$
H=\left\{\alpha \in H^{2}(X, \mathbb{R}): c_{1} \cdot \alpha=0\right\}
$$

does not meet the Kähler cone of $X_{k}$ unless $k \geq 9$. It follows that if $k \leq 8$, every Kähler class $[\omega]$ satisfies $c_{1}(X) \cdot[\omega]>0$. Since this number represents, up to a factor of $4 \pi$, the integral of the scalar curvature over $X$, it follows that any CSC Kähler metric on $X_{k}$ must be positive, whatever the Kähler class.

Now we turn to the case $k \geq 9$. In [12], it was proved that there exist zero CSC Kähler metrics on $X_{k}$ in this case (the same result was previously known for $k \geq 14$ [7]). In the next result, we obtain CSC Kähler metrics of either sign:

Theorem B. There exists a nine-point blow-up $X$ of $\mathbb{C P}^{1} \times \mathbb{C P}^{1}$ with no non-trivial holomorphic vector field and the following properties:

(i) there is a scalar-flat Kähler metric on $X$;

(ii) for every $c \in \mathbb{R}$ and $\varepsilon>0$, there exists a metric of constant scalar curvature $\omega$ on $X$ such that

$$
\left|\frac{[\omega] \cdot c_{1}(X)}{\sqrt{[\omega]^{2}}}-c\right| \leq \varepsilon .
$$

In addition, any further blow-up of $X$ admits metrics with the same properties.

As mentioned for Theorem $\mathrm{A}$, the Kähler classes of the metrics provided in Theorem $\mathrm{B}$ will be explicit, up to a parameter $\varepsilon$, once the tools used to construct the metrics are introduced at Section 2 and Theorem $\mathrm{C}$

Part (i) of this theorem was proved in [12]. It plays in this discussion an analogous role to the one played by Kähler-Einstein metrics in Theorem $\mathrm{A}$ and corresponds to $c=c^{\text {sfk }}=0$. Again, the new content of this theorem is that there are metrics of constant scalar curvature that represent Kähler classes arbitrarily far from the hyperplane $H$ inside $H^{2}(X, \mathbb{R})$. 
Remark. Kähler metrics of constant scalar curvature admit a good perturbation theory: if the class $[\omega]$ is represented by a CSC Kähler metric, then the same is true for all sufficiently close Kähler classes, and also for all small pertubations of the complex structure, at least if $X$ carries no non-zero holomorphic vector fields [8]. This applies to the KE metrics on $X_{6}$ and $X_{7}$ to give non-Einstein CSC metrics in all classes sufficiently close to $c_{1}(X)$. It also applies to give CSC Kähler metrics of either sign on $X_{9}$, by perturbing a known zero CSC metric. It seems very difficult, however, to extend this perturbation theory to give more global results like Theorems A or B A possible strategy to get larger pertubations could be to follow the lines of a recent beautiful work of Chen-LeBrun-Weber [4], based on continuity method, that enables them to construct a particular extremal Kähler metric conformal to an Einstein metric on $\mathbb{C P}^{2}$ blown up twice.

\subsection{Strategy}

Theorems A and B will be deduced from an extension of the constructions invented in [12] and the recent gluing theorem of Arezzo-Pacard [1] for CSC Kähler metrics. The zero CSC Kähler metrics in [12] were obtained by resolving the singularities of a zero CSC orbifold. The gluing theorem in that work applied only to produce zero CSC Kähler metrics. The results of [1] allow us to work with CSC orbifolds of non-zero scalar curvature, which are, however, constructed in almost exactly the same way as in [12]. Indeed, as will be explained below, these orbifolds are twisted products of Riemann surfaces, where now the scalar curvature of the factors can be chosen arbitrarily.

Remark. In §9(i) of their paper, Arezzo and Pacard make the following construction: take a copy of $X_{k}$ with no holomorphic vector field and Tian's Kähler-Einstein metric (so $k \leq 7)$. Their gluing theorem gives a positive CSC Kähler metric on the blow-up of $X_{k}$, that is to say, on $X_{k+1}$. If $k=7$, then this proves Theorem A in the particular case $c=2$, $k=8$.

We should point out that in contrast to this approach, our construction does not use any result about the existence of Kähler-Einstein metrics on del Pezzo surfaces and may be considered to be more elementary for this reason.

Remark. The gluing theorem of [1] is not specific to complex dimension 2. Accordingly, in $\$ 6$ we give some new but rather special higher-dimensional examples of negative CSC Kähler metrics.

\section{The factory}

We review the construction of scalar-flat Kähler orbifold metrics of [12] and adapt it to the case of Kähler metrics of constant scalar curvature. 


\subsection{Orbifold Riemann surfaces}

We start with a closed Riemann surface $\bar{\Sigma}$ of genus $g$ with a finite set of orbifold points $P_{1}, \ldots, P_{k}$, with local ramified cover of orders $q_{1}, \ldots, q_{k}>1$. Recall first the description of the fundamental group of the punctured Riemann surface $\Sigma=\bar{\Sigma} \backslash\left\{P_{j}\right\}$ :

$$
\pi_{1}(\Sigma)=\left\langle a_{1}, b_{1}, \ldots, a_{g}, b_{g}, l_{1}, \ldots, l_{k}:\left[a_{1}, b_{1}\right]\left[a_{2}, b_{2}\right] \ldots\left[a_{g}, b_{g}\right] l_{1} \ldots l_{k}=1\right\rangle .
$$

Here the $a_{j}$ and $b_{j}$ are standard generators of $\pi_{1}(\bar{\Sigma})$ and $l_{j}$ is (the homotopy class of) a small loop around $P_{j}$. The orbifold fundamental group is defined by

$$
\pi_{1}^{\mathrm{orb}}(\bar{\Sigma})=\pi_{1}(\Sigma) / G
$$

where $G$ is the normal subgroup of $\pi_{1}(\Sigma)$ generated by $l_{1}^{q_{1}}, \ldots, l_{k}^{q_{k}}$.

The orbifold Euler characteristic is defined by

$$
\chi^{\text {orb }}(\bar{\Sigma}):=\chi^{\text {top }}(\bar{\Sigma})-\sum_{j=1}^{k}\left(1-\frac{1}{q_{j}}\right)
$$

The question of whether $\bar{\Sigma}$ carries a CSC orbifold Kähler metric was considered by Troyanov [14]. Let us call an orbifold Riemann surface good if its orbifold universal cover admits a compatible Kähler metric of CSC $\kappa_{1}$, say. By the Gauss-Bonnet theorem (applied to the surface with boundary obtained by removing a small disc around each of the $P_{j}$ ), if $\bar{\Sigma}$ is good, then the sign of $\kappa_{1}$ is the same as the sign of $\chi^{\text {orb }}(\bar{\Sigma})$. However, not every orbifold Riemann surface is good: the tear-drop, which is $S^{2}$ with one orbifold point of any order $\geq 2$, is simply connected but admits no compatible metric of constant curvature.

The following summarizes the facts we shall need if the orbifold Euler characterstic is non-positive:

Proposition 2.1.1. The orbifold Riemann surface $\bar{\Sigma}$ is always good if

$$
\chi^{\mathrm{orb}}(\bar{\Sigma}) \leq 0
$$

Such a $\bar{\Sigma}$ carries no non-trivial holomorphic vector fields if $\chi^{\text {orb }}(\bar{\Sigma})<0$ or if $\chi^{\text {orb }}(\bar{\Sigma})$ $=0$ and there is at least one orbifold point.

Proof. The existence of metrics of constant scalar curvature is contained in [14]. The statement about holomorphic vector fields follows either by lifting such a field to the universal cover or from the orbifold version of the Hopf index theorem: a holomorphic vector field must vanish at each of the orbifold points.

For good orbifolds with positive orbifold Euler characteristic, the universal cover must be $\mathbb{C P}^{1}$ and the orbifold fundamental group must be one of the finite subgroups of $\mathrm{SO}(3)$ : 
Proposition 2.1.2. Let $\bar{\Sigma}$ be a good orbifold with $\chi^{\mathrm{orb}}(\bar{\Sigma})>0$. Then $\bar{\Sigma}$ is biholomorphic to $\mathbb{C P}^{1} / \Gamma$, where $\Gamma$ is a finite subgroup of $\mathrm{SO}(3)$. There are either two marked points of the same order $q \geq 2$ or three marked points with orders $\{2,2, q\},\{2,3,3\}$, $\{2,3,4\},\{2,3,5\}$. The corresponding groups are cyclic of order $q$, dihedral, tetrahedral, octahedral, or icosahedral respectively.

Furthermore, $\bar{\Sigma}$ carries no non-trivial holomorphic vector fields if and only if $G$ is not cyclic.

Proof. We need only discuss the last part. The Euler vector field $\left(z \partial_{z}\right.$ in affine coordinates) descends to the quotient if $G$ is the cyclic group acting with fixed points at $z=0$ and $z=\infty$. On the other hand, no non-trival holomorphic vector field on the sphere can have three zeros, so none of the other orbifolds can admit such vector fields.

\subsection{Desingularization of orbifold ruled surfaces}

From now on we assume that $\bar{\Sigma}$ is a good orbifold Riemann surface and we endow $\bar{\Sigma}$ with an orbifold Kähler metric $\omega_{\kappa_{1}}^{\prime}$ of constant curvature $\kappa_{1}$. Note that, just as for ordinary Riemann surfaces, we have

$$
\bar{\Sigma}=\widetilde{\Sigma} / \pi_{1}^{\mathrm{orb}}(\bar{\Sigma})
$$

where the fundamental group acts by isometries on the universal cover $\widetilde{\Sigma}$ which is given by either $\mathbb{H}, \mathbb{E}$ or $\mathbb{C P}^{1}$, endowed with its metric $\widetilde{\omega}_{\kappa_{1}}^{\prime}$ of constant curvature $\kappa_{1}$.

Let $\omega_{\kappa_{2}}^{\prime \prime}$ be the Fubini-Study metric with curvature $\kappa_{2}>0$, on $\mathbb{C P}^{1}$. Let $\rho: \pi_{1}^{\text {orb }}(\bar{\Sigma})$ $\rightarrow \mathrm{SU}(2) / \mathbb{Z}_{2}$ be a homomorphism. Then $\pi_{1}^{\text {orb }}(\bar{\Sigma})$ acts isometrically on $\widetilde{\Sigma} \times \mathbb{C P}^{1}$ endowed with the product metric $\widetilde{\omega}_{\kappa_{1}}^{\prime}+\omega_{\kappa_{2}}^{\prime \prime}$. Therefore it gives rise to a Kähler metric $\bar{\omega}_{\rho}$ of constant scalar curvature $s=2\left(\kappa_{1}+\kappa_{2}\right)$ on the orbifold quotient

$$
\bar{M}_{\rho}=\bar{\Sigma} \times_{\rho} \mathbb{C P}^{1}=\left(\widetilde{\Sigma} \times \mathbb{C P}^{1}\right) / \pi_{1}^{\text {orb }}(\bar{\Sigma}) .
$$

As in the smooth case, the de Rham cohomology $H_{D R}^{2}\left(\bar{M}_{\rho}\right)$ of the orbifold ruled surface $\bar{M}_{\rho} \rightarrow \bar{\Sigma}$ is given by $H_{D R}^{2}\left(\bar{M}_{\rho}\right)=\mathbb{R} h \oplus \mathbb{R} f$ where $f$ is the Poincaré dual of a generic fibre $F$, and we have $h \cdot f=1$ and $h^{2}=f^{2}=0$.

By construction of the metric, its orbifold Kähler class $\bar{\Omega} \in H_{D R}^{2}\left(\bar{M}_{\rho}\right)$ satisfies $\bar{\Omega} \cdot f=4 \pi / \kappa_{2}$, and $\bar{\Omega} \cdot h=2 \pi \chi^{\mathrm{orb}}(\bar{\Sigma}) / \kappa_{1}$ (by Gauss-Bonnet). We introduce $v_{1}=$ $2 \pi \chi^{\operatorname{orb}}(\bar{\Sigma}) / \kappa_{1}$, which is the volume of the metric $\omega_{\kappa_{1}}^{\prime}$. Similarly put $v_{2}=4 \pi / \kappa_{2}$. It is now easy to pin down the orbifold Kähler class:

$$
\bar{\Omega}=v_{1} f+v_{2} h .
$$

The idea now is to apply the results of [1] to obtain CSC Kähler metrics on the minimal resolution of singularities, $\widehat{M}_{\rho}$, say, of $\bar{M}_{\rho}$. We note that the constant $s$ can be chosen arbitrarily if $\bar{\Sigma}$ is hyperbolic, but must be positive otherwise. Here is the full statement, which is parallel to Theorem D of [12]. 
Theorem C. Let $\bar{\Sigma}$ be a good compact orbifold Riemann surface as above, carrying no non-trivial holomorphic vector fields. Suppose that $\rho: \pi_{1}^{\mathrm{orb}}(\bar{\Sigma}) \rightarrow \mathrm{SU}(2) / \mathbb{Z}_{2}$ is a homomorphism that is irreducible in the sense that the induced action of $\pi_{1}^{\mathrm{orb}}(\bar{\Sigma})$ fixes no point of $\mathbb{C P} \mathbb{P}^{1}$. Equip $\bar{M}_{\rho}$ with a twisted product metric of CSC $s$ as above.

Then, for all $\varepsilon>0$, the minimal resolution $\widehat{M}_{\rho} \stackrel{\pi}{\rightarrow} \bar{M}_{\rho}$ carries a CSC Kähler metric $\hat{g}$ with scalar curvature $\hat{s}$ such that $|\hat{s}-s|<\varepsilon$. Moreover, the Kähler class $\hat{\Omega}$ of $\hat{g}$ satisfies $\left\|\hat{\Omega}-\pi^{*} \bar{\Omega}\right\|<\varepsilon$ (where $\|\cdot\|$ is any Euclidean norm fixed a priori on $\left.H_{D R}^{2}\left(\widehat{M}_{\rho}\right)\right)$.

In addition, any further blow-up of $\widehat{M}_{\rho}$ carries a CSC Kähler metric with analogous properties.

Proof. We have already equipped $\bar{M}_{\rho}$ with a Kähler orbifold metric of constant scalar curvature $s$. On the other hand, for each finite cyclic subgroup $G$ of $U_{2}$ with the property that $G$ acts freely on $S^{3}$, there is an asymptotically locally euclidean zero CSC Kähler metric on the minimal resolution [3, 12]. If $s=0$, we are in the situation of [12], so we may as well assume that $s \neq 0$. We must check that the hypotheses of [1] are satisfied, i.e. that $\bar{M}_{\rho}$ has no non-trivial holomorphic vector fields. But this follows as in [12], for we have placed ourselves in the situation where the base $\bar{\Sigma}$ has no non-zero holomorphic vector fields.

Hence, we can indeed apply [1] to obtain CSC Kähler metrics $g$ on $\widehat{M}_{\rho}$. For sufficiently small choices of the gluing parameter, $g$ is very close to $g_{\rho}$, and the scalar curvature of $g$ will be very close to the number $s$.

To get the last statement, we again apply [1], this time gluing a copy of the Burns metric at a smooth point.

Remark. The term "minimal" applied here is really unfortunate. According to its usual meaning in complex surface theory, it means that there are no rational curves of selfintersection -1 . In this case, however, the proper transform of any singular fibre of $\bar{M}_{\rho}$ is always a (-1)-curve! The term "minimal" is justified by the fact that we are taking the minimal resolution of each of the orbifold singularities separately. Only after doing so, is it possible to blow down the $(-1)$-curves.

\section{Stability of parabolic ruled surfaces}

In order to make the results of the previous section more useful, we need a way to generate representations $\rho: \pi_{1}^{\text {orb }}(\bar{\Sigma}) \rightarrow \operatorname{SU}(2) / \mathbb{Z}_{2}$. A way to do this was introduced in [12], through the notion of a (stable) parabolic ruled surface. We recall the main definitions here:

A geometrically ruled surface $\breve{M}$ is by definition a minimal complex surface obtained as $\breve{M}=\mathbb{P}(E)$, where $E \rightarrow \widehat{\Sigma}$ is a holomorphic vector bundle of rank 2 over a smooth Riemann surface $\widehat{\Sigma}$. The induced map $\pi: \widehat{M} \rightarrow \widehat{\Sigma}$ is called the ruling.

A parabolic structure on $\widetilde{M}$ consists of the following data:

- A finite set of distinct points $P_{1}, \ldots, P_{n}$ in $\widehat{\Sigma}$;

- for each $j$, a choice of point $Q_{j} \in F_{j}=\pi^{-1}\left(P_{j}\right)$; 
- for each $j$, a choice of weight $\alpha_{j} \in(0,1) \cap \mathbb{Q}$.

A geometrically ruled surface with a parabolic structure will be called a parabolic ruled surface.

If $S \subset \widetilde{M}$ is a holomorphic section of $\pi$, we define its slope to be

$$
\mu(S)=S^{2}+\sum_{Q_{j} \notin S} \alpha_{j}-\sum_{Q_{j} \in S} \alpha_{j}
$$

we say that a parabolic ruled surface is stable if for every holomorphic section $S$, we have $\mu(S)>0$.

\subsection{Iterated blow-up of a parabolic ruled surface}

Let $\widetilde{M}$ be a parabolic ruled surface. We shall now define a multiple blow-up $\Phi: \widehat{M} \rightarrow \widetilde{M}$ which is canonically determined by the parabolic structure of $\bar{M}$.

In order to simplify the notation, suppose that the parabolic structure on $\widetilde{M}$ is reduced to a single point $P \in \widehat{\Sigma}$; let $Q$ be the corresponding point in $F=\pi^{-1}(P)$ and let $\alpha=p / q$ be the weight, where $p$ and $q$ are two coprime integers, $0<p<q$. Denote the Hirzebruch-Jung continued fraction expansion of $\alpha$ by

$$
\frac{p}{q}=\frac{1}{e_{1}-\frac{1}{e_{2}-\cdots \frac{1}{e_{l}}}}
$$

define also

$$
\frac{q-p}{q}=\frac{1}{e_{1}^{\prime}-\frac{1}{e_{2}^{\prime}-\cdots \frac{1}{e_{m}^{\prime}}}}
$$

These expansions are unique if, as we shall assume, the $e_{j}$ and $e_{j}^{\prime}$ are all $\geq 2$.

We give here a construction of the iterated blow-up $\widehat{M}$ : the fibre $F$ has self-intersection 0 . The first step is to blow up $Q$, to get a diagram of the form

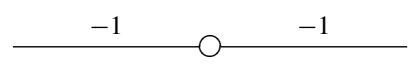

By blowing up the intersection point of these two curves we get the diagram

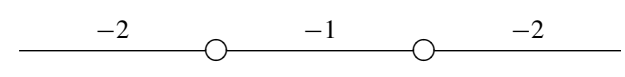


Then we perform an iterated blow-up of one of the two intersections of the only $(-1)$ curve in the diagram. Given $\alpha=p / q$, there is a unique way (cf. [12, Proposition 2.1.1]) to choose at each step which point has to be blown up in order to get the following diagram:

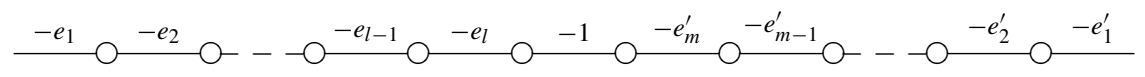

where the $\left(-e_{1}\right)$-curve is the proper transform of the original fibre $F$.

More generally, if $\bar{M}$ has more parabolic points, we perform the same operation for every point and get a corresponding iterated blow-up $\Phi: \widehat{M} \rightarrow \breve{M}$.

\subsection{The theorem of Mehta-Seshadri}

We now give a practical method of using Theorem C. Given a parabolic ruled surface $\breve{M} \rightarrow \widehat{\Sigma}$, we deduce a orbifold Riemann surface $\bar{\Sigma}$ by introducing an orbifold singularity of order $q_{j}$ at every parabolic point $P_{j} \in \widehat{\Sigma}$ of weight $p_{j} / q_{j}$. As a corollary of the MehtaSeshadri theorem [10], we have the following proposition.

Proposition 3.2.1. Let $\breve{M} \rightarrow \widehat{\Sigma}$ be a parabolically stable ruled surface. Then there exists an irreducible representation $\rho: \pi_{1}^{\text {orb }}(\bar{\Sigma}) \rightarrow \mathrm{SU}(2) / \mathbb{Z}_{2}$ such that $\widehat{M} \simeq \widehat{M}_{\rho}$, where $\widehat{M}$ is the iterated blow-up of the parabolic ruled surface $\bar{M}$ as described in Section 3.1 and $\widehat{M}_{\rho}$ is the smooth resolution of the orbifold $\bar{M}_{\rho}$ defined in Section 2

Proof. This is a direct consequence of [12, Theorem 3.3.1].

In conclusion we can reformulate Theorem $\mathrm{C}$ as follows.

Theorem D. Let $\breve{M} \rightarrow \widehat{\Sigma}$ be a parabolically stable ruled surface, with parabolic weights $\alpha_{j}=p_{j} / q_{j}$ where $p_{j}$ and $q_{j}$ are positive coprime integers. Let $\bar{\Sigma}$ be the orbifold ruled surface deduced from $\widehat{\Sigma}$ according to the parabolic structure. Then the conclusions (i) and (ii) of Theorem $C$ hold with $\widehat{M}_{\rho}$ replaced by $\widehat{M}$.

\section{The goods: proof of Theorems $A$ and $B$}

\subsection{A stable parabolic bundle}

Let $\bar{\Sigma}$ be $\mathbb{C P}^{1}$ with three orbifold points $P_{1}, P_{2}, P_{3}$, each of weight 2 . Then the orbifold Euler characterstic is $1 / 2$ and $\bar{\Sigma}$ is good, and we can equip $\bar{\Sigma}$ with a metric of CSC $\kappa_{1}>0$. Now, we consider the ruled surface $\pi: \mathbb{C P}^{1} \times \mathbb{C P}^{1} \rightarrow \mathbb{C P}^{1}$ where $\pi$ is, say, the projection on the first factor. We pick a point $Q_{j}$ in the fibre $\pi^{-1}\left(P_{j}\right)$, and give it weight $\alpha_{j}=1 / 2$.

Lemma 4.1.1. For a generic choice of points $Q_{1}, Q_{2}, Q_{3}$, the parabolic ruled surface $\breve{M} \rightarrow \mathbb{C P}^{1}$ defined above is parabolically stable.

Proof. We just need to arrange that two points $Q_{j}$ never belong to the same constant section of $\bar{M} \rightarrow \mathbb{C P}^{1}$. 


\subsection{Conclusion}

The iterated blow-up $\widehat{M}$ of Theorem D in this case consists of performing two blow-ups in each fibre, so that $\widehat{M}$ is a six-point blow-up of $\mathbb{C P}^{1} \times \mathbb{C P}^{1}$. Thus this result yields positive CSC Kähler metrics on $X_{6}$. By adjusting $\kappa_{1}$ and $\kappa_{2}$ and taking the gluing parameter to be small enough, we can get any positive value of $c$ in 1.1.

To deal with $X_{7}$ and $X_{8}$, we can use the last part of Theorem $\mathrm{C}$ to perform further blow-ups on the previous example. Alternatively, we can replace one of the parabolic weights by $1 / 3$ or $1 / 4$ in the above. The complex surface $X_{8}$ can also be constructed by adding a fourth parabolic point $P_{4}$, and taking all weights equal to $1 / 2$. It is readily seen that for generic choices of the points $Q_{j}$, the resulting parabolic ruled surfaces are stable.

Remark. We deliberately chose $\kappa_{1}$ and $\kappa_{2}$ so as to get CSC metrics that are far from being Einstein. However, if we take $\kappa_{1}=\kappa_{2}$ then it seems possible that with a little more work this gluing construction might be adapted to give the KE metrics for (certain complex structures on) $X_{6}$ and $X_{7}$ !

It would also be interesting to try to extend the analysis to allow $\bar{M}_{\rho}$ to carry nontrivial holomorphic vector fields. This problem is actually a work in progress [11], based on a refinement of a recent gluing theorem of Arezzo-Pacard [2] in the orbifold case.

\subsection{Proof of Theorem $B$}

The proof follows the same lines as in [12] (or as above), taking $\bar{\Sigma}$ to be $\mathbb{C P} \mathbb{P}^{1}$ with four orbifold points of order 2, 2, 2, 3. The orbifold Euler characteristic is $-1 / 6$ and so we can equip $\bar{M}_{\rho}$ with a CSC Kähler metric with normalized scalar curvature $c_{1} \cdot[\omega] / \sqrt{[\omega]^{2}}$ equal to any given real number. Applying Theorem $\mathrm{D}$, we get the result claimed in Theorem $B$

\section{Explicit representations}

Our use of the theory of stability to generate representations of the orbifold fundamental group results in a certain loss of explicitness-one of the merits of gluing constructions is that they give "nearly" explicit metrics, in the sense that for small values of the gluing parameter, the glued metric is close to the original one. From this point of view, it seems desirable to try to make explicit these representations, at least in a few examples, and this section is devoted to such a task.

\subsection{Platonic orbifold surfaces}

Let $\Gamma$ be one of the finite subgroups of $\mathrm{SO}(3)$ as in Proposition 2.1.2 Then there is a canonical orbifold $\bar{M}=\mathbb{C P}^{1} \times \mathbb{C P}^{1} / \Gamma$, where $\Gamma$ acts diagonally on the product. If $\Gamma$ is not cyclic, then this orbifold will not support any non-trivial holomorphic vector fields and the resolution will carry a positive CSC Kähler metric. 


\subsection{Representations of the fundamental group of the four-fold punctured sphere}

Let $\bar{\Sigma}$ be the orbifold Riemann surface used in the proof of Theorem B We shall construct representations of the orbifold fundamental group of $\bar{\Sigma}$. Let the points be denoted $P_{j}$, with orders 2, 2, 2, 3 respectively, and let $l_{j}$ denote the homotopy class of a small loop around $P_{j}$. The orbifold fundamental group is

$$
\pi_{1}^{\mathrm{orb}}(\bar{\Sigma})=\left\langle l_{1}, l_{2}, l_{3}, l_{4}: l_{1} l_{2} l_{3} l_{4}=l_{1}^{2}=l_{2}^{2}=l_{3}^{2}=l_{4}^{3}=1\right\rangle .
$$

Identifying $\mathrm{SU}(2)$ with the group of unit quaternions, we write down a family of representations $\pi_{1}^{\text {orb }} \rightarrow \mathrm{SU}(2) / \mathbb{Z}_{2}$ :

$$
\begin{aligned}
& \rho\left(l_{1}\right)= \pm i, \quad \rho\left(l_{2}\right)= \pm j, \quad \rho\left(l_{3}\right)= \pm k \exp (\pi(i \cos \phi+j \sin \phi) / 3), \\
& \rho\left(l_{4}\right)= \pm \exp (-\pi(i \cos \phi+j \sin \phi) / 3) .
\end{aligned}
$$

Denote by $R_{1}, R_{2}, R_{3}$ and $R_{4}$ the corresponding rotations of $\mathbb{R}^{3}$. Then $R_{1}, R_{2}$ and $R_{3}$ are half-turns, while $R_{4}$ is a $2 \pi / 3$-rotation (see Figure 1 .

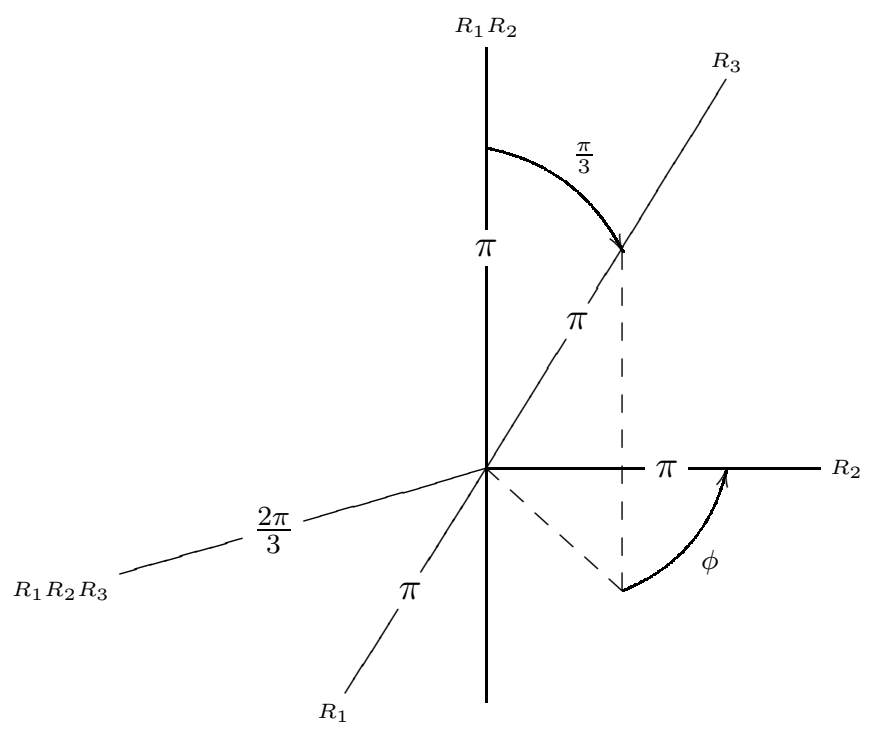

Fig. 1. A representation of the fundamental group of the four-fold punctured sphere.

This representation is irreducible and so gives rise to a CSC Kähler orbifold $\bar{M}_{\rho}$. The corresponding minimal resolution $\widehat{M}_{\rho}$ is the nine-point blow-up of a minimal ruled surface $\breve{M} \rightarrow \mathbb{C P}^{1}$. We do not know which ruled surface this is for general $\phi$, but if $\phi=0$, we sketch an argument explaining why $\bar{M}$ is isomorphic to $\mathbb{C P}^{1} \times \mathbb{C P}^{1}$, so that the scalar-flat Kähler manifold $\widehat{M}_{\rho}$ is isomorphic to a nine-point blow-up of $\mathbb{C P}^{1} \times \mathbb{C P}^{1}$. 
Taking $\phi=0$ from now on, we note that the $x$-axis is stable under the action of $\rho$ : it is fixed pointwise by $R_{1}$ and $R_{4}$ and flipped by $R_{2}$ and $R_{3}$. Set $Q_{1}=(1,0,0), Q_{2}=$ $(-1,0,0)$ and consider the Riemann surface

$$
S:=\left(\mathbb{C P}^{1} \backslash\left\{P_{j}\right\}\right) \times_{\rho}\left\{Q_{1}, Q_{2}\right\} .
$$

This sits in $\bar{M}$, and its closure $\bar{S}$ is a two-fold ramified cover $p: \bar{S} \rightarrow \bar{\Sigma}$. In $\bar{S}$, there are two orbifold singularities sitting over each of $P_{1}$ and $P_{4}$; on the other hand, the cover is ramified over $P_{2}$ and $P_{3}$ and the corresponding points in $\bar{S}$ are smooth. Thus $\bar{S}$ is an orbifold version of $\mathbb{C P}^{1}$, with two singular points of weight 2 and two singular points of weight 3 . Denote by $\bar{N}$ the associated orbifold ruled surface, and by $\widehat{N}$ its minimal resolution.

Downstairs in $\bar{M}$ there are eight orbifold singularities, say,

$$
A_{j}, B_{j} \in \bar{\pi}^{-1}\left(P_{j}\right) .
$$

Upstairs in $\bar{N}, P_{1}$ and $P_{4}$ have been double-covered by points $P_{1}^{ \pm}, P_{4}^{ \pm}$and there are accordingly singularities

$$
A_{1}^{ \pm}, B_{1}^{ \pm} \in \bar{\pi}^{-1}\left(P_{1}^{ \pm}\right), \quad A_{4}^{ \pm}, B_{4}^{ \pm} \in \bar{\pi}^{-1}\left(P_{4}^{ \pm}\right) .
$$

The double cover is ramified over $P_{2}$ and $P_{3}$, so the points $A_{2}^{\prime}, A_{3}^{\prime}, B_{2}^{\prime}, B_{3}^{\prime}$ corresponding to $A_{2}, A_{3}, B_{2}, B_{3}$ are smooth. There is a commutative diagram

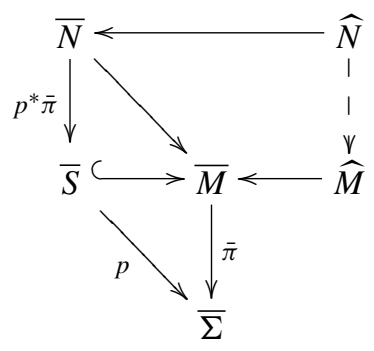

where the dashed arrow is a meromorphic map which becomes a smooth ramified double cover if the points $A_{2}^{\prime}, A_{3}^{\prime}, B_{2}^{\prime}, B_{3}^{\prime}$ are blown up.

The reason for passing to $\bar{N}$ is that it contains two parallel sections $s_{1}$ and $s_{2}$, defined by $s_{1}\left(Q_{1}\right)=Q_{1}, s_{1}\left(Q_{2}\right)=Q_{2}$ and $s_{2}\left(Q_{1}\right)=Q_{2}, s_{2}\left(Q_{2}\right)=Q_{1}$; let $\bar{S}_{j}$ denote the closure of $s_{j}(\bar{S})$ in $\bar{N}$. Because the $s_{j}$ are parallel, $\bar{S}_{j}^{2}=0$. By a computation that we omit, the proper transforms $\widehat{S}_{j}$ of $\bar{S}_{j}$ in $\widehat{N}$ satisfy $\widehat{S}_{1} \cdot \widehat{S}_{2}=0$ and

$$
\widehat{S}_{1}^{2}=\widehat{S}_{2}^{2}=-2
$$

(see Figure2).

After a sequence of five blow-downs in a suitable order in $\widehat{M}$ (corresponding to ten blow-downs on $\widehat{N}$ ) we obtain the configuration of curves shown in Figure 3 . 


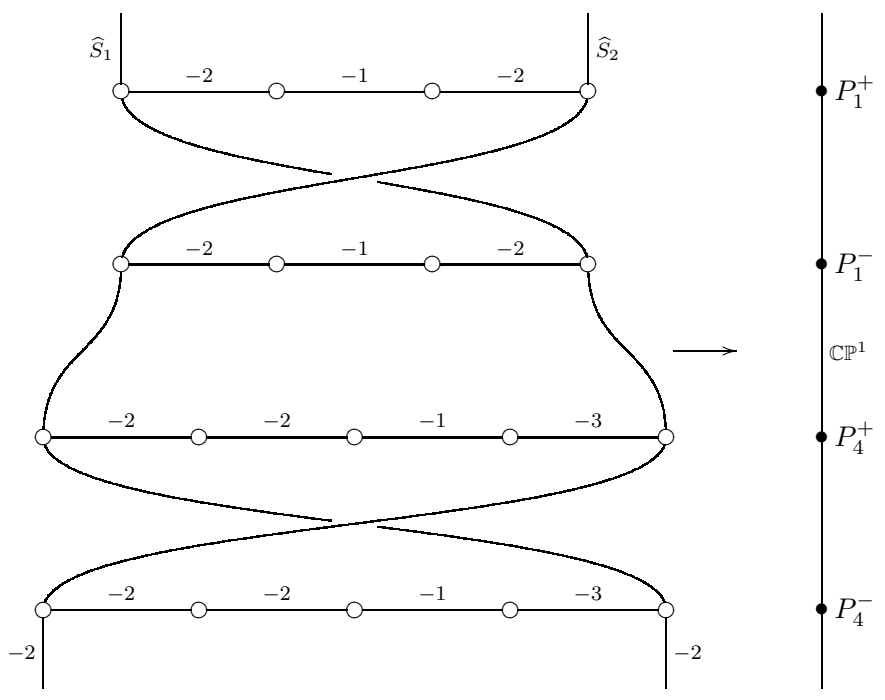

Fig. 2. Configuration of curves in $\widehat{N}$.

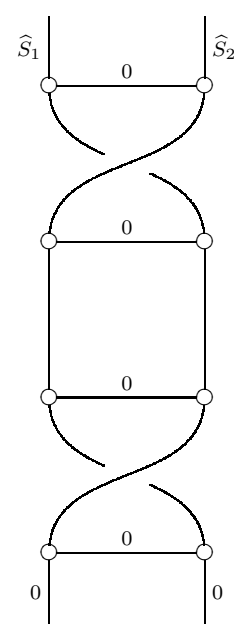

Fig. 3. Ten-point blowdown of $\widehat{N}$.

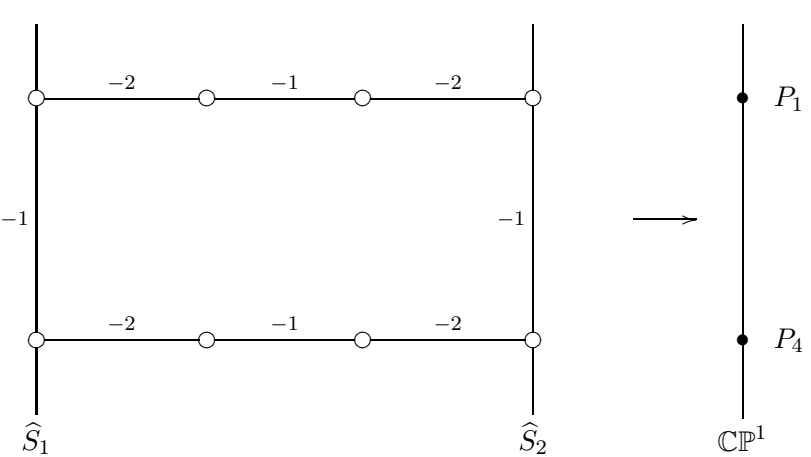

Fig. 4. $\widehat{M}$ with five $(-1)$-curves blown down.

It is a minimal ruled surface with a divisor $D$ satisfying $D \cdot F=1$ and $D^{2}=0$, where $F$ is the class of a generic fibre. Therefore it is isomorphic to $\mathbb{C P}^{1} \times \mathbb{C P}^{1}$. Thus $\widehat{M}$ is a five-point blow-up of the minimal resolution of $\left(\mathbb{C P}^{1} \times \mathbb{C P}^{1}\right) / \mathbb{Z}_{2}$, where the action of $\mathbb{Z}_{2}$ rotates each factor by an angle $\pi$.

It is easy to see that the latter complex surface is a four-point iterated blow-up of $\mathbb{C P}^{1} \times \mathbb{C P}^{1}$. Consider the proper transforms $\widehat{S}_{1}$ of $\bar{S}_{1}=\left(0 \times \mathbb{C P}^{1}\right) / \mathbb{Z}_{2}$ and $\widehat{S}_{2}$ of $\bar{S}_{2}=$ $\left(\mathbb{C P}^{1} \times 0\right) / \mathbb{Z}_{2}$. Then we have $\widehat{S}_{1} \cdot \widehat{S}_{2}=0$ and $\widehat{S}_{1}^{2}=\widehat{S}_{2}^{2}=-1$. Hence we have the 
configuration of curves shown in Figure 4 and after four blow-downs in a suitable order, we obtain $\mathbb{C P}^{1} \times \mathbb{C P}^{1}$.

\subsection{Representations of the fundamental group of the punctured torus}

Let $\mathbb{T}$ be a compact elliptic curve with one marked point $P$ of weight 2 , and let $\bar{\Sigma}$ stand for the corresponding orbifold Riemann surface. In terms of standard generators,

$$
\pi_{1}^{\mathrm{orb}}(\bar{\Sigma})=\left\langle a, b, l:[a, b] l=l^{2}=1\right\rangle .
$$

We define a representation $\rho: \pi_{1}^{\text {orb }}(\bar{\Sigma}) \rightarrow \mathrm{SU}(2) / \mathbb{Z}_{2}$ as follows:

$$
\rho(a)= \pm e^{\pi k / 4} i, \quad \rho(b)= \pm j, \quad \rho(l)= \pm k .
$$

The corresponding rotations $R_{a}, R_{b}, R_{l}$ are shown in Figure 5

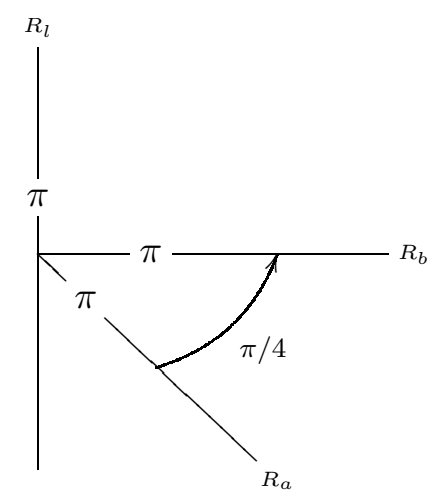

Fig. 5. A representation of the punctured torus.

Denote by $\bar{M}$ the CSC Kähler orbifold ruled surface arising from this representation, by $\widehat{M}$ the minimal resolution, and by $\breve{M} \rightarrow \mathbb{T}$ a minimal model for $\widehat{M}$.

Then we have

Proposition 5.3.1. The surface $\widehat{M}$ is a double blow-up of $\breve{M}$, and carries a CSC Kähler metric. On the other hand, no smooth blow-down of $\widehat{M}$ admits a CSC Kähler metric.

We give a sketch of the proof of this. The existence of a metric on $\widehat{M}$ is ensured by Theorem C. Consider, then, the non-existence statement. The geometrically ruled surface $\breve{M}$ cannot admit a CSC Kähler metric for $c_{1}^{2}=0$, but $c_{1} \neq 0$. In $\widehat{M}$ the only curve that can be blown down smoothly is in the fibre over $P$. Denote by $X$ this blow-down.

As in the previous example, we can pass to a double cover by remembering the two points on the unit sphere on the axis of $R_{l}$. We obtain a double cover $\bar{N}$ of $\bar{M}$ and a corresponding minimal resolution $\widehat{N}$. By considering the geometry of this double cover, 
one can show that the double cover $X^{\prime}$ of $X$ is a two-point blow-up of $\mathbb{P}\left(L_{1} \oplus L_{2}\right) \rightarrow \mathbb{T}$, where the points now lie on the sections corresponding to $L_{1}$ and $L_{2}$. Such an $X^{\prime}$ does not admit a CSC Kähler metric by [9, Prop. 3.1], and so $X$ cannot admit a CSC Kähler metric either.

\section{Higher-dimensional examples}

Since the results of [1] apply in all dimensions, it is natural to try to extend the foregoing results to higher dimensions. The main problem here is that very little appears to be known about the following basic

Question 6.1. If $G$ is a finite subgroup of $U_{m}$ acting freely on $\mathbb{C}^{m} \backslash 0$, and $X \rightarrow \mathbb{C}^{m} / G$ is a resolution of singularities, does there exist an asymptotically locally euclidean zero CSC Kähler metric on $X$ ?

Joyce [6] proved that if $X$ can be chosen to be a crepant resolution (i.e. $c_{1}(X)=0$ ), then $X$ does carry an ALE Ricci-flat metric. In dimensions 2 and 3, this is the case if and only if $G$ is (conjugate to) a subgroup of $\mathrm{SU}(m)$. In higher dimensions, however, it is not known in general which singularities $\mathbb{C}^{m} / G$ admit crepant resolutions.

The other class of examples come from explicit constructions of Kähler metrics on total spaces of line bundles, as described, for example, in [5] which contains an extensive survey of the literature. The following result follows easily from the methods of that paper, and is probably well known to many. We sketch the proof in order to make this paper self-contained.

Theorem 6.2. If $G=\left\{1, \omega, \ldots, \omega^{k-1}\right\}$, where $\omega=e^{2 \pi i / k}$ then there is a resolution $X$ of $\mathbb{C}^{m+1} / G$ that admits an asymptotically locally euclidean zero CSC Kähler metric.

Proof. We note that for this group $G$, we can take $X$ to be the total space of $\mathcal{O}(-k) \rightarrow$ $\mathbb{C P}^{m}$. Indeed, the total space of $\mathcal{O}(-1)$ can be represented as $U / \mathbb{C}^{*}$, where

$$
U=\left\{\left(w, z_{0}, \ldots, z_{m}\right) \in \mathbb{C} \times\left(\mathbb{C}^{m} \backslash 0\right)\right\},
$$

the action of $\mathbb{C}^{*}$ is given by

$$
t \cdot\left(w, z_{0}, \ldots, z_{m}\right)=\left(t^{-1} w, t z_{0}, \ldots, t z_{m}\right),
$$

and the map

$$
\beta\left(w, z_{0}, \ldots, z_{m}\right) \mapsto\left(w z_{0}, w z_{1}, \ldots, w z_{m}\right)
$$

is an invariant description of the blow-up of the origin of $\mathbb{C}^{m+1}$. The action of the group $G$ lifts to the action

$$
\omega^{r}\left(w, z_{0}, z_{1}, \ldots, z_{m}\right)=\left(\omega^{r} w, z_{0}, z_{1}, \ldots, z_{m}\right)
$$

on $U$, with quotient equal to the total space of $\mathcal{O}(-k)$. 
Following an Ansatz that goes back to Calabi, we seek a Kähler metric on $X$ of the form

$$
\omega_{f}=\omega_{0}+i \partial \bar{\partial} f(t)
$$

where $\omega_{0}$ is the standard Kähler form on $\mathbb{C P}^{m}$ and $t$ is the logarithm of the fibre-distance function.

To be more explicit, we work with affine coordinates $\left(z_{1}, \ldots, z_{m}\right)$ on $\mathbb{C P}^{m}$ so that

$$
\omega_{0}=(i / 2) \partial \bar{\partial} \log \left(1+\left|z_{1}\right|^{2}+\cdots+\left|z_{m}\right|^{2}\right) .
$$

In the same coordinates, the fibre metric on $\mathcal{O}(-k)$ is just given by

$$
h^{k}=\left(1+\left|z_{1}\right|^{2}+\cdots+\left|z_{m}\right|^{2}\right)^{k} .
$$

Denote by $w$ a holomorphic fibre coordinate, and $\operatorname{set} z=\log w$ so that

$$
2 t=z+\bar{z}+k \log h .
$$

We have

$$
i \partial \bar{\partial} t=(i k / 2) \partial \bar{\partial} \log h=k \omega_{0}
$$

so that 6.1 can be written

$$
\omega_{f}=\left(1+k f^{\prime}(t)\right) \omega_{0}+f^{\prime \prime}(t) i \partial t \wedge \bar{\partial} t
$$

As explained in [5], it is better to pass to momentum coordinates, so we introduce

$$
\tau=f^{\prime}(t) \quad \text { and } \quad \varphi(\tau)=f^{\prime \prime}(t)
$$

The advantage of this is that the scalar curvature is given by a very simple formula which we now derive. First of all,

$$
\omega_{f}^{m+1}=(1+k \tau)^{m} h^{-m-1} \varphi(\tau) \psi \wedge \bar{\psi}
$$

where $\psi$ is a holomorphic $(m+1)$-form, so that

$$
\begin{aligned}
\rho\left(\omega_{f}\right) & =-i \partial \bar{\partial} \log \left[(1+k \tau)^{m} h^{-m-1} \varphi(\tau)\right] \\
& =2(m+1) \omega_{0}-i \partial \bar{\partial} \log \left[(1+k \tau)^{m} \varphi(\tau)\right] .
\end{aligned}
$$

Now for any function of $u(\tau)$,

$$
i \partial \bar{\partial} u(\tau)=k \varphi u^{\prime} \omega_{0}+\varphi\left(\varphi u^{\prime}\right)^{\prime} i \partial t \wedge \bar{\partial} t
$$

where prime denotes differentiation with respect to $\tau$. Applying this with

$$
u(\tau)=m \log (1+k \tau)+\log \varphi(\tau)
$$

we obtain

$$
\rho\left(\omega_{f}\right)=\left[2(m+1)-k \varphi u^{\prime}\right] \omega_{0}-\varphi\left(\varphi u^{\prime}\right)^{\prime} i \partial t \wedge \bar{\partial} t
$$


The scalar curvature $\sigma\left(\omega_{f}\right)$ is given (up to a factor of 2) by

$$
\sigma\left(\omega_{f}\right) \omega_{f}^{m+1}=(m+1) \rho\left(\omega_{f}\right) \wedge \omega_{f}^{m},
$$

which leads to the final formula

$$
\sigma\left(\omega_{f}\right)=\frac{2 m(m+1)}{1+k \tau}-\frac{1}{(1+k \tau)^{m}} \frac{\mathrm{d}^{2}}{\mathrm{~d} \tau^{2}}\left((1+k \tau)^{m} \varphi(\tau)\right) .
$$

Thus $\sigma\left(\omega_{f}\right)=0$ if

$$
(1+k \tau)^{m} \varphi(\tau)=\frac{2}{k^{2}}(1+k \tau)^{m+1}+a \tau+b
$$

where $a$ and $b$ are constants of integration. The boundary conditions for smoothness of the metric at the zero section are

$$
\varphi(0)=0, \quad \varphi^{\prime}(0)=2,
$$

which gives

$$
P(\tau):=(1+k \tau)^{m} \varphi(\tau)=\frac{2}{k^{2}}\left((1+k \tau)^{m+1}+(k-m-1)(1+k \tau)+m-k\right) .
$$

Geometrically, we have repackaged the complex line bundle $X$ as the total space of an $S^{1}$-bundle over $\mathbb{C P}^{m} \times[0, \infty)_{\tau}$; to check that $\omega_{f}$ is really a metric on this $S^{1}$-bundle we need that $\varphi(\tau)>0$ for all $\tau \in(0, \infty)$. To see that this is the case, note that

$$
P^{\prime}(\tau)=0 \quad \text { if and only if } \quad(1+k \tau)^{m}=1-\frac{k}{m+1},
$$

and this latter equation cannot be satisfied if $\tau>0$. Since $P^{\prime}(0)=2$, we see that $P^{\prime}(\tau)>0$ for all $\tau>0$, and since $P(0)=0$ we have $P(\tau)>0$ for all $\tau>0$ as claimed. Thus our zero CSC Kähler metric on $X$ corresponds to the function

$$
\varphi(\tau)=\frac{2}{k^{2}}\left((1+k \tau)+(k-m-1)(1+k \tau)^{1-m}+(m-k)(1+k \tau)^{-m}\right) .
$$

Finally, we check the asymptotics of this metric. For this, we return to the blow-up map at the beginning of the proof. In the present coordinates, this is

$$
x_{0}=w^{1 / k}, \quad x_{1}=w^{1 / k} z_{1}, \ldots, x_{m}=w^{1 / k} z_{m},
$$

where $\left(x_{0}, x_{1}, \ldots, x_{m}\right)$ are standard linear coordinates on $\mathbb{C}^{m+1}$, and the fractional power of $w$ corresponds to the passage from $\mathcal{O}(-k)$ to its $k$-fold cover $\mathcal{O}(-1)$. The standard Kähler form on $\mathbb{C}^{m+1}$ is

$$
\eta=\frac{i}{2} \partial \bar{\partial}\left(\left|x_{0}\right|^{2}+\cdots+\left|x_{m}\right|^{2}\right)=\frac{i}{2} \partial \bar{\partial}\left(|w|^{2 / k} h\right)=\frac{i}{2} \partial \bar{\partial} e^{2 t / k} .
$$


Thus we can write $\eta$ in the form 6.1

$$
\eta=\omega_{f_{0}}
$$

where

$$
f_{0}(t)=\frac{1}{2} e^{2 t / k}-t / k
$$

Denoting the corresponding variables (6.3) by $\tau_{0}$ and $\phi_{0}$, we have

$$
1+k \tau_{0}=e^{2 t / k}, \quad \varphi_{0}\left(\tau_{0}\right)=\frac{2}{k^{2}}\left(1+k \tau_{0}\right) .
$$

We now use the relation

$$
d t=\frac{d \tau_{0}}{\varphi_{0}\left(\tau_{0}\right)}=\frac{d \tau}{\varphi(\tau)}
$$

to find an asymptotic expansion of $f(t)$ for large $t$. Indeed, if we put

$$
x=1+k \tau_{0}, \quad y=1+k \tau, \quad y=x(1+u(x))
$$

we see that 6.17) gives the equation

$$
x \frac{\mathrm{d} u}{\mathrm{~d} x}=(k-m-1) x^{-m}(1+u)^{1-m}+(m-k) x^{-m-1}(1+u)^{-m}
$$

for $u$. This has a unique solution with $u(x) \rightarrow 0$ as $x \rightarrow \infty$, and we have

$$
u(x) \sim \frac{m+1-k}{m} x^{-m}+\frac{k-m}{m+1} x^{-m-1}+\cdots \quad \text { as } x \rightarrow \infty .
$$

Using the relations (6.18) and 6.16, we find

$$
1+k f^{\prime}(t)=e^{2 t / k}+\frac{m+1-k}{m} e^{2(1-m) t / k}+\frac{k-m}{m+1} e^{-2 m t / k}+\cdots
$$

and so

$$
f=|x|^{2}+\frac{m+1-k}{m}|x|^{2-2 m}+\frac{k-m}{m+1}|x|^{-2 m}+\cdots
$$

where the second term on the right must be replaced by $k(2-k) \log |x|$ if $m=1$. This completes the proof that $\omega_{f}$ is ALE.

We give two applications of this result:

Theorem 6.3. Let $S_{1}, \ldots, S_{m}$ be compact Riemann surfaces, each admitting a holomorphic involution $\iota_{j}$ with isolated fixed points. Suppose further that none of the $S_{j}$ is $\mathbb{C P}^{1}$, and that at least one is hyperbolic. Let $\iota=\left(\iota_{1}, \ldots, \iota_{m}\right)$ be the product involution on $S=S_{1} \times \cdots \times S_{m}$. Then the minimal resolution $\widehat{M}$ of $S / \iota$ admits a negative CSC Kähler metric.

In addition, any further blow-up of $\widehat{M}$ at finitely many points carries a CSC Kähler metric with negative scalar curvature. 
Proof. Equip $S_{j}$ with a metric of constant curvature $\kappa_{j}$. The product metric on $S$ is not Ricci-flat because there is at least one hyperbolic factor. Moreover, the orbifold $S / \iota$ has no non-zero holomorphic vector fields. This is clear if each factor is hyperbolic, for then $S$ has no non-zero holomorphic fields. If one or more factors in the product are flat, then $M$ does have non-zero holomorphic vector fields, but none of them is preserved by $\iota$. Hence for any choice $\left(\kappa_{1}, \ldots, \kappa_{m}\right), S / \iota$ is an unobstructed CSC Kähler orbifold, and every singularity is modelled on $\mathbb{C}^{m} /( \pm 1)$. Theorem 6.2 with $k=2$, now gives a resolution of this singularity, and applying the desingularization theorem of [1] gives the result. The scalar curvature of $\widehat{M}$ is negative since the sum of the $\kappa_{j}$ 's is negative.

For the last statement, the CSC Kähler metric on the blow-up is obtained by gluing the ALE metric provided by Theorem 6.2, with $k=1$.

Theorem 6.4. Let $\bar{\Sigma}$ be a good compact orbifold Riemann surface as defined in Section 2.1. carrying no non-trivial holomorphic vector fields, and with orbifold points of order 2 only. Suppose that $\rho: \pi_{1}^{\text {orb }}(\bar{\Sigma}) \rightarrow \mathrm{SU}(2) / \mathbb{Z}_{2}$ is a homomorphism that is irreducible in the sense that the induced action of $\pi_{1}^{\text {orb }}(\bar{\Sigma})$ fixes no point of $\mathbb{C P}^{1}$.

Using the diagonal action of $\pi_{1}(\bar{\Sigma})$ on $\left(\mathbb{C P}^{1}\right)^{m}$ induced by $\rho$, define the twisted product $\bar{M}_{\rho}=\bar{\Sigma} \times_{\rho}\left(\mathbb{C P}^{1}\right)^{m}$. This is a complex orbifold with singularities modelled on $\mathbb{C}^{m+1} /\{ \pm 1\}$. Equip $\bar{M}_{\rho}$ with a twisted product metric of CSC. Then the minimal resolution $\widehat{M}_{\rho} \rightarrow \bar{M}_{\rho}$ carries a CSC Kähler metric.

In addition, any further blow-up of $\widehat{M}_{\rho}$ at finitely many points carries a CSC Kähler metric.

Proof. Again we will use the ALE spaces of Theorem 6.2, with $k=2$ and $k=1$ (for the last statement). In order to use the gluing theorem of [1], the only thing that needs to be checked is that $\bar{M}_{\rho}$ does not carry any non-trivial holomorphic vector field. The argument is a straightforward generalization of the one given in [12] in the case $m=1$.

Remark. In contrast to the previous theorem, by adjusting the curvatures of the base and the fibre here, in the case where $\chi^{\text {orb }}(\bar{\Sigma})<0$, we can arrange for the scalar curvature of $\widehat{M}_{\rho}$ to be positive, zero, or negative.

Acknowledgments. We thank Claudio Arezzo, Frank Pacard and Denis Auroux for stimulating discussions.

The first author was partly supported by NSF grant \# DMS-0305130.

The second author was partly supported by a Leverhulme research fellowship and an EPSRC small grant.

\section{References}

[1] Arezzo, C., Pacard, F.: Blowing up and desingularizing constant scalar curvature Kähler manifolds. Acta Math. 196, 179-228 (2006) Zbl 1123.53036 MR 2275832

[2] Arezzo, C., Pacard, F.: Blowing up and desingularizing constant scalar curvature Kähler manifolds II. math.DG/0504115

[3] Calderbank, D. M. J., Singer, M. A.: Einstein metrics and complex singularities. Invent. Math. 156, 405-443 (2004) Zbl 1061.53026 MR 2052611 
[4] Chen, X., LeBrun, C., Weber, B.: On conformally Kähler, Einstein manifolds. J. Amer. Math. Soc. 21, 1137-1168 (2008) MR 2425183

[5] Hwang, A. D., Singer, M. A.: A momentum construction for circle-invariant Kähler metrics. Trans. Amer. Math. Soc. 354, 2285-2325 (2002) Zbl 0987.53032 MR 1885653

[6] Joyce, D. D.: Compact Manifolds with Special Holonomy. Oxford Univ. Press, Oxford, 2000 Zbl 1027.53052 MR 1787733

[7] Kim, J., LeBrun, C., Pontecorvo, M.: Scalar-flat Kähler surfaces of all genera. J. Reine Angew. Math. 486, 69-95 (1997) Zbl 0876.53044 MR 1450751

[8] LeBrun, C., Simanca, S. R.: Extremal Kähler metrics and complex deformation theory. Geom. Funct. Anal. 4, 298-336 (1994) Zbl 0801.53050 MR 1274118

[9] LeBrun, C., Singer, M.: Existence and deformation theory for scalar-flat Kähler metrics on compact complex surfaces. Invent. Math. 112, 273-313 (1993) Zbl 0793.53067 MR 1213104

[10] Mehta, V. B., Seshadri, C. S.: Moduli of vector bundles on curves with parabolic structures. Math. Ann. 248, 205-239 (1980) Zbl 0454.14006 MR 0575939

[11] Rollin, Y., Singer, M.: Kähler surfaces, and parabolic polystability. Submitted.

[12] Rollin, Y., Singer, M.: Non-minimal scalar-flat Kähler surfaces and parabolic stability. Invent. Math. 162, 235-270 (2005) Zbl 1083.32021 MR 2199006

[13] Tian, G.: On Calabi's conjecture for complex surfaces with positive first Chern class. Invent. Math. 101, 101-172 (1990) Zbl 0716.32019 MR 1055713

[14] Troyanov, M.: Prescribing curvature on compact surfaces with conical singularities. Trans. Amer. Math. Soc. 324, 793-821 (1991) Zbl 0724.53023 MR 1005085 